\title{
The Effects of the Prone Position on the Physiological Function in Healthy Students
}

\author{
Seiko Miyata ${ }^{1}$, Akiko Noda ${ }^{1, *}$, Yuki Fujii ${ }^{2}$, Atsuko Maekawa ${ }^{3}$, Masato Okuda ${ }^{4}$, Chie Nakazaki $^{2}$, \\ Hironao Otake ${ }^{4}$, Seiichi Nakata ${ }^{5}$, Fumihiko Yasuma ${ }^{6}$, Yasuo Koike ${ }^{7}$, Kaoru Shimokata ${ }^{8}$, Tsutomu \\ Nakashima $^{4}$, Shigeaki Hinohara ${ }^{9}$ \\ ${ }^{I}$ Department of Biomedical Sciences, Chubu University, Kasugai, Japan \\ ${ }^{2}$ Department of Medical Technology, Nagoya University School of Health Sciences, Nagoya, Japan \\ ${ }^{3}$ Department of Nursing, Nagoya University School of Health Science, Nagoya, Japan \\ ${ }^{4}$ Department of Otorhinolaryngology, Nagoya University Graduate School of Medicine, Nagoya, Japan \\ ${ }^{5}$ Department of Otorhinolaryngology, Second Hospital, Fujita Health University School of Medicine, Nagoya, Japan \\ ${ }^{6}$ Department of Internal Medicine, Suzuka National Hospital, Suzuka, Japan \\ ${ }^{7}$ Department of Clinical Engineering, Chubu University, Kasugai, Japan \\ ${ }^{8}$ Department of Lifelong Sports for Health, Chubu University, Kasugai, Japan \\ ${ }^{9}$ St. Luke's International Hospital, Tokyo, Japan
}

\begin{abstract}
The rapid improvement in blood oxygenation has been observed in patients with acute respiratory failure placed in a prone position. The gravitational effect relieves upper airway narrowing in the prone position, resulting in an improvement in sleep quality. This study examined the effect of the prone position on autonomic nerve activity and cardiovascular function. Seven healthy adults (mean age $25.2 \pm 10.6$ years) enrolled in this study. The heart rate variability as a marker of autonomic activity, lactate value as a maker of fatigue, vascular function (brachial-ankle pulse wave velocity; baPWV), and blood pressure in the supine and prone position were evaluated. The heart rate variability was calculated using an electrocardiogram to measure the RR interval. The high-frequency (HF) and low-frequency /high-frequency (LF/HF) were calculated on baseline, after 30-min in the supine and prone position. The ratio of baseline HF to that after 30-min significantly increased in the prone position than the supine position. The ratio of baseline LF/HF to that after 30min in the prone position significantly decreased compared to the supine position. Meanwhile, the ratio of the baseline LF to that after 30-min did not show any significant difference in the prone position in comparison to the supine position. There were no significant differences on lactate value, baPWV and blood pressure change. The prone position might therefore have a beneficial effect on the autonomic function.
\end{abstract}

Keywords: parasympathetic activity, sympathetic activity, prone positioning, heart rate variability.

\section{INTRODUCTION}

Recent advances in critical care medicine and rehabilitation have demonstrated the significance of prone position therapy, which has been utilized for the treatment of patients with acute respiratory distress syndrome (ARDS) $[1,2]$ and acute respiratory failure [3] in intensive care unit. The function of the diaphragm on spontaneous- and artificialrespiration was studied using 3-dimensional computer tomography in healthy adults, in which the diaphragmatic muscle tone during sleep were diminished in the supine position and it was increased in the prone position [4]. An improvement of $\mathrm{PaO}_{2}$ by $10 \mathrm{~mm} \mathrm{Hg}$ or more was observed in patients with

*Address correspondence to this author at the Department of Biomedical Sciences, Chubu University. 1200, Matsumoto-cho, Kasugai-shi, Aichi 4878501, Japan; Tel: +81-568-51-9906; Fax: +81-568-51-5370;

E-mail: anoda@isc.chubu.ac.jp
ARDS after 30-min in the prone position; however, returning the patients to the supine position reversed the improvement in gas exchange [5]. Respiratory care in prone position in a patient with respiratory failure might improve oxygenation efficiency and ventilation-perfusion mismatch [6].

A possible mechanism of the prone position to improve oxygenation even in healthy people as well as treating the hypoxemic patients with ARDS might be the correction in mismatch of the ventilation-perfusion ratio through the positional relationship [2]. An upper airway is obstructive when the negative pressure on respiration overcomes the upper airway patency during sleep [7]. Another mechanism of the prone position to improve oxygenation and to stabilize respiration might be relieving upper airway narrowing during sleep [8]. Moreover, the autonomic activity during prone position might affect oxygenation or respiration. For example, in patients with coronary artery disease, the parasympathetic activity in the supine position was significantly lower 
Table 1. All Measurements at Baseline and after 30-Min in the Supine and Prone Position

\begin{tabular}{|c|c|c|c|}
\hline & Baseline & Supine Position & Prone Position \\
\hline \hline Autonomic activity & & & $962.4 \pm 995.5$ \\
\hline HF $\left(\mathrm{ms}^{2}\right)$ & $868.4 \pm 680.4$ & $674.0 \pm 565.4$ & $1546.0 \pm 2395.0$ \\
\hline LF $\left(\mathrm{ms}^{2}\right)$ & $1334.2 \pm 1661.7$ & $33.7 \pm 15.7$ & $49.2 \pm 20.1$ \\
\hline normalized HF & $35.5 \pm 15.3$ & $59.3 \pm 22.0$ & $44.6 \pm 23.2$ \\
\hline normalized LF & $57.7 \pm 21.5$ & $3.8 \pm 5.7$ & $1.3 \pm 1.2$ \\
\hline LF/HF & $2.9 \pm 3.5$ & $2.8 \pm 1.9$ & $3.4 \pm 2.3$ \\
\hline Lactic acid (mmol/l) & $4.9 \pm 5.0$ & & $1049.0 \pm 244.3$ \\
\hline baPWV & & $990.2 \pm 171.3$ & $1086.8 \pm 174.1$ \\
\hline Right $(\mathrm{cm} / \mathrm{s})$ & $986.2 \pm 155.1$ & $1063.5 \pm 87.2$ & \\
\hline Left $(\mathrm{cm} / \mathrm{s})$ & $1024.4 \pm 163.6$ & & \\
\hline
\end{tabular}

HF: high-frequency, LF: low-frequency, LF/HF: low-frequency divided with high-frequency, baPWV: brachial-ankle pulse wave velocity. The normalized HF and LF were calculated as $=\mathrm{HF} /(\mathrm{HF}+\mathrm{LF}) \times 100$ or $=\mathrm{LF} /(\mathrm{HF}+\mathrm{LF}) \times 100$.

than that in the prone position [9]. Since the effects of the prone position on autonomic activity and cardiovascular function are mostly unknown, we investigated them in healthy volunteers.

\section{MATERIALS AND METHODS}

\section{Subjects}

In seven healthy students (mean age $25.2 \pm 10.6$ years), the autonomic activity, fatigue, vascular function, and blood pressure in the supine and prone position were evaluated. All measurements at baseline were performed in the supine position after 15 minutes bed rest, then the subjects were randomly allocated to rest on a bed in the supine or prone position for 30 minutes. The study protocol was approved by the appropriate institutional review committee, and the subjects provided written consent to participation after being informed in detail of the purpose and methods of the study.

\section{Autonomic Activity Evaluation}

Holter electrocardiogram (recorded with a NASA lead; placed on the upper segment of the sternum and on the ensiform process) was recorded to measure the RR interval. A heart rate variability (Memcalc/Win GMS Co. Tokyo, Japan) was calculated from the 5 minutes segments of RR interval records. The low-frequency (LF) band $(0.04$ to $0.15 \mathrm{~Hz}$ ), high-frequency $(\mathrm{HF})$ band $(0.15$ to $0.40 \mathrm{~Hz})$ and the ratio of $\mathrm{LF}$ to $\mathrm{HF}(\mathrm{LF} / \mathrm{HF})$ were calculated on baseline and in the supine and prone position after 30-min. The LF and HF components were measured in $\mathrm{ms}^{2}$ and are expressed as power and normalized $\mathrm{HF}$ and $\mathrm{LF}$ were calculated as = $\mathrm{HF} /(\mathrm{HF}+\mathrm{LF}) \times 100$ or $=\mathrm{LF} /(\mathrm{HF}+\mathrm{LF}) \times 100$. From the clinical view point, it has been generally accept that the HF component mainly reflects parasympathetic activity, LF reflects parasympathetic and sympathetic activity and LF/HF reflects sympathetic activity [10].

\section{Fatigue Evaluation}

Lactate in blood was measured using Lactate Pro (LT1710; Arkray Inc., Kyoto, Japan). The blood of $5 \mu 1$ or more was drawn from the end of the forefinger by the injection needle, then put on the check chip that contained the reagent, and the lactic acid value was measured. Measurement was at baseline and after 30-min in the supine and prone position.

\section{Brachial-Ankle Pulse Wave Velocity Measurement}

The brachial-ankle pulse wave velocity (baPWV) was measured using a volume-plethysmographic apparatus (PWV/ABI, Omron Colin, Tokyo, Japan) according to methods previously reported $[11,12]$. The device recorded baPWV, blood pressure, ECG and heart sounds simultaneously. ECG electrodes were placed on both wrists, and a heart sound microphone was placed on the left sternal border. The cuffs to measure baPWV were wrapped around both the upper arms and ankles, and connected to a plethysmographic sensor that determined volume pulse form. Volume waveforms were stored for a sampling time of $10 \mathrm{sec}-$ onds with automatic gain analysis and quality adjustment. The time delay from the ascending point of the brachial artery waveform to the ascending point of each tibial artery waveform $(\Delta \mathrm{Tba})$ was determined. The distance of each segment (Lb-La) was automatically calculated based on the patient's height and was derived from statistical studies. Then, the baPWV was calculated using the formula: $\mathrm{baPWV}=\mathrm{La}-$ $\mathrm{Lb} / \Delta \mathrm{Tba}(\mathrm{cm} / \mathrm{s})$. The baPWV was measured at baseline and after 30-min in supine and prone position.

\section{Statistical Analysis}

Data are presented as the means $\pm \mathrm{SD}$, and were analyzed by paired $t$-test. All analyses were performed with the Statview statistical software package (SAS Institute Inc; Cary, NC). A $P$ value of $<0.05$ was considered to be statistically significant.

\section{RESULTS}

The HF measured by the spectral analysis of heart rate variability increased at 30-min after the prone position, and $\mathrm{LF} / \mathrm{HF}$ tended to decrease at 30-min after the prone position. There was no significant difference in the LF between at baseline and at 30-min after the prone position. The normalized HF increased at 30-min after the prone position, and normalized LF tended to decrease at 30-min after the prone position (Table 1). 
Table 2. The Comparison of the Change Ratio of Heart Rate Variability between Supine and Prone Position

\begin{tabular}{|c|c|c|}
\hline & Supine Position & Prone Position \\
\hline \hline HF & $1.0 \pm 1.0$ & $2.5 \pm 2.1^{*}$ \\
\hline LF & $1.6 \pm 1.3$ & $1.2 \pm 0.6$ \\
\hline normalized HF & $0.9 \pm 0.1$ & $1.5 \pm 0.5^{*}$ \\
\hline normalized LF & $1.0 \pm 0.1$ & $0.7 \pm 0.1^{*}$ \\
\hline LF/HF & $1.2 \pm 0.2$ & $0.5 \pm 0.2^{*}$ \\
\hline
\end{tabular}

$*: P$ value $<0.05$, vs supine position. HF: high-frequency, LF: low-frequency, LF/HF: low-frequency divided with high-frequency. The normalized HF and LF were calculated as = $\mathrm{HF} /(\mathrm{HF}+\mathrm{LF}) \times 100$ or $=\mathrm{LF} /(\mathrm{HF}+\mathrm{LF}) \times 100$. The change ratio of heart rate variability was calculated as $=$ the value at 30 -min after supine or prone position/the value at baseline.

The ratio of baseline HF to that 30-min after significantly increased in the prone position than the supine position $(2.5 \pm 2.1$ vs $1.0 \pm 1.0, P=0.041)$. There was no significant difference in the ratio of baseline LF to that 30-min after between in the supine and prone position. The ratio of baseline normalized HF to that 30-min after significantly increased in the prone position than the supine position $(1.5 \pm 0.5$ vs $0.9 \pm 0.1, P=0.041)$. The ratio of baseline normalized LF to that 30-min after significantly decreased in the prone position than the supine position $(0.7 \pm 0.1$ vs $1.0 \pm 0.1, P=0.013)$. The ratio of baseline LF/HF to that 30-min after significantly decreased in the prone position than the supine position $(0.5 \pm 0.2$ vs $1.2 \pm 0.2, P=0.025)$ (Table 2 ).

There was no significant difference in the value of lactic acid between the baseline and 30-min after in either the prone or supine position. There was no significant difference in the baPWV between baseline and 30-min after in either the prone or supine position (Table 1).

\section{DISCUSSION}

The ratio of HF significantly increased and the ratio of LF/HF also significantly decreased in the prone position compared with those in the supine position. HF and LF/HF did not show any significant differences between supine and prone position. There were no significant differences on lactate value, baPWV and blood pressure. Our findings suggest that the prone position might have a significant effect on parasympathetic activity even in healthy volunteers.

This study demonstrated that the HF reflecting the parasympathetic activity increased and the LF/HF reflecting the sympathetic activity [10] decreased in the prone position in comparison with the supine position. Several mechanisms might be involved in the beneficial effect of the prone position on autonomic modulation. A volume overload in right atrium causes momentary control of the discharge in the noradrenergic activity, resulting in a change in the autonomic activity [13]. The prone position may play an important role in the change of autonomic activity thorough the volume receptor in the right atrium. Turning prone position from supine position eliminates the compressive force of the heart on dorsal lung regions. This change will lower the inspiratory pressure required to obtain maximal air-space recruitment; and lower the end-expiratory pressure required to maintain maximal air-space recruitment [2]. Arterial blood oxygen tension was increased in the prone position because of an correction in ventilation and perfusion mismatching, which is secondary to increased homogeneity of the ventila- tion distribution and increased correlation of regional ventilation and perfusion $[14,15]$ The volume receptor in the right atrium, lessening the compression of the lungs by the heart and the improvement in oxygenation in the prone position, is the possible mechanism of the improvement in cardiac vagal modulation.

Previous studies demonstrated the relationship between sleep problems and autonomic function. Insomnia patients with objectively determined short sleep duration reduced parasympathetic activity as indicated by decreased HF [16]. The increased chemoreflex gain in patients with obstructive sleep apnea resulted in tonic chemoreflex activation even during normoxia, with consequent increased sympathetic activity [17]. Increased sympathetic activity relates with morbidity and mortality of cardiovascular disease [18]. Thus, our finding that the prone position enhanced the parasympathetic activity in healthy volunteers suggests that the prone sleep position might be beneficial to prevent developing cardiovascular diseases for patients with sleep disorders and sleep disordered-breathing.

There was no significant difference between supine and prone positions in the lactic acid reflecting the muscle fatigue [19]. The baPWV would identify patients with a high risk of amalgamation of cardiovascular disease related to arteriosclerosis $[20,21]$, however, we found that there was no significant difference in the baPWV between the supine and prone position. Moreover, there was no significant difference in the blood pressure between the supine and prone position. Daily sleep position might influence sleep quality. Therefore, further evaluation will be necessary to determine the effect of the prone position on the cardiovascular function and sleep quality.

\section{CONCLUSION}

The ratio of $\mathrm{HF}$ at baseline to 30-min after prone position significantly increased and the ratio of baseline LF/HF to that 30 -min after prone position was also intentionally reduced compared to those on supine position. The prone positioning might therefore have a beneficial effect on the autonomic function, and the further evaluation, long term randomized control trial and case-control study, is needed to clarify the long term effect of the prone position on cardiovascular function.

\section{LIST OF ABBREVIATIONS}

ARDS $=$ acute respiratory distress syndrome 
baPWV $=$ brachial-ankle pulse wave velocity

$\mathrm{HF} \quad=$ high-frequency

$\mathrm{LF} \quad=$ low-frequency

$\mathrm{Lb}-\mathrm{La}=$ distance of each brachial to ankle

$\Delta$ Tba $=$ the time delay from the ascending point of the brachial artery waveform to the ascending point of each tibial artery waveform

\section{CONFLICT OF INTEREST}

There are no conflicts of interest to disclose, and the authors declare no relationships with industry.

\section{ACKNOWLEDGEMENT}

Declared none.

\section{REFERENCES}

[1] Mancebo J, Fernández R, Blanch L, et al. A multicenter trial of prolonged prone ventilation in severe acute respiratory distress syndrome. Am J Respir Crit Care Med 2006; 173: 1233-9.

[2] Albert RK, Hubmayr RD. The prone position eliminates compression of the lungs by the heart. Am J Respir Crit Care Med 2000; 161: $1660-5$

[3] Gattinoni L, Tognoni G, Pesenti A, et al. Prone-Supine Study Group: effect of prone positioning on the survival of patients with acute respiratory failure. N Engl J Med 2001; 345: 568-73.

[4] Krayer S, Rehder K, Vettermann J, Didier EP, Ritman EL. Position and motion of the human diaphragm during anesthesia-paralysis. Anesthesiology 1989; 70: 891-8.

[5] Pappert D, Rossaint R, Slama K, Grüning T, Falke KJ. Influence of positioning on ventilation-perfusion relationships in severe adult respiratory distress syndrome. Chest 1994; 106: 1511-6.

[6] Hemmila MR, Napolitano LM. Severe respiratory failure: advanced treatment options. Crit Care Med 2006; 34: S278-90.

[7] Budhiraja R, Parthasarathy S, Drake CL, et al. Early CPAP use identifies subsequent adherence to CPAP theory. Sleep 2007; 30: 320-4.

[8] Ingman T, Nieminen T, Hurmerinta K. Cephalometric comparison of pharyngeal changes in subjects with upper airway resistance syndrome or obstructive sleep apnoea in upright and supine positions. Eur J Orthod 2004; 26: 321-6.

[9] Yang JL, Chen GY, Kuo CD. Comparison of effect of 5 recumbent positions on autonomic nervous modulation in patients with coronary artery disease. Circ J 2008; 72: 902-8.

[10] Task Force of the European Society of Cardiology and the North American Society of pacing and electrophysiology. heart rate variability: standards of measurement, physiological interpretation and clinical use. Circulation 1996; 93: 1043-65.

[11] Munakata M, Ito N, Nunokawa T, Yoshinaga K. Utility of automated brachial ankle pulse wave velocity measurements in hypertensive patients. Am J Hypertens 2003; 16: 653-7.

[12] Yamashina A, Tomiyama H, Takeda K, et al. Validity, reproducibility, and clinical significance of noninvasive brachial-ankle pulse wave velocity measurement. Hypertens Res 2002; 25: 359-64.

[13] Murakawa Y, Ajiki K, Usui M, Yamashita T, Oikawa N, Inoue H. Parasympathetic activity is a major modulator of the circadian variability of heart rate in healthy subjects and in patients with coronary artery disease or diabetes mellitus. Am Heart J 1993; 126: 108-14.

[14] Lamm WJE, Graham MM, Albert RK. Mechanism by which the prone position improves oxygenation in acute lung injury. Am J Respir Crit Care Med 1994; 150: 184-93.

[15] Mure M, Domino KB, Lindahl SGE, Hlastala MP, Altemeier WA, Glenny RW. Regional ventilation-perfusion distribution is more uniform in the prone position. J Appl Physiol 2000; 88: 1076-83.

[16] Spiegelhalder K, Fuchs L, Ladwig J, et al. Heart rate and heart rate variability in subjectively reported insomnia. J Sleep Res 2011; 20: 137-45.

[17] Narkiewicz K, van de Borne PJ, Montano N, Dyken ME, Phillips BG, Somers VK. Contribution of tonic chemoreflex activation to sympathetic activity and blood pressure in patients with obstructive sleep apnea. Circulation 1998; 97: 943-5.

[18] Thayer JF, Yamamoto SS, Brosschot JF. The relationship of autonomic imbalance, heart rate variability and cardiovascular disease risk factors. Int J Cardiol 2010; 141: 122-31.

[19] Surenkok O, Kin-Isler A, Aytar A, Gültekin Z. Effect of trunkmuscle fatigue and lactic acid accumulation on balance in healthy subjects. J Sport Rehabil 2008; 17: 380-6.

[20] Yamashina A, Tomiyama H, Arai T, et al. Brachial-ankle pulse wave velocity as a marker of atherosclerotic vascular damage and cardiovascular risk. Hypertens Res 2003; 26: 615-22.

[21] Blacher J, Guerin AP, Pannier B, Marchais SJ, Safar ME, London GM. Impact of aortic stiffness on survival in end-stage renal disease. Circulation 1999; 99: 2434-9.

(C) Miyata et al.; Licensee Bentham Open.

This is an open access article licensed under the terms of the Creative Commons Attribution Non-Commercial License (http://creativecommons.org/licenses/by-nc/3.0/) which permits unrestricted, non-commercial use, distribution and reproduction in any medium, provided the work is properly cited. 